\title{
A case study of the impact on postprandial plasma glucose based on the 14- day sensor device reliability (GH-Method: math-physical medicine)
}

Gerald C. Hsu

EclaireMD Foundation, USA

\author{
"Correspondence author \\ Gerald C Hsu \\ EclaireMD Foundation \\ USA
}

Submitted : 12 May 2020 ; Published : 27 May 2020

\section{Introduction}

In this paper, the author analyzed the impact on glucose based on the 14-day sensor device reliability during a four-month period from 6/2/2019 - 10/12/2019 using GH-Method: mathphysical medicine.

\section{Methods}

During a period of 13 months (5/5/2018 - 6/1/2019), the author used a 10-day glucose sensor device (Sensor) that was applied to his upper left arm to collect 74 glucose data per day. On $6 / 2 / 2019$, he switched to a newer device with a longer lifespan (14-day) of glucose measurements. However, he immediately noticed some unusual higher or lower glucose values recorded with this new sensor device. Through this 14day sensor sub-period, he did not change his lifestyle details, i.e. diet and exercise habits, dramatically. In order to keep the integrity of his medical research, during the entire period of 5/5/2018 - 10/12/2019 (526 days), he has kept two parallel glucose measurements using both finger-piercing and test strip (Finger) along with Sensor. To collect a meaningful size of data, he completed the three-month window before starting his 14-day sensor data examination and analysis work. The reason is that the average lifespan of red blood cells is approximately three months and the associated timespan of $\mathrm{HbA} 1 \mathrm{C}$ is about three months.

In order to better understand the above-mentioned "unusually higher or lower" sensor glucose activities, he began with a study of PPG's formation and its physical characteristics and behaviors. After completing this task, he can then derive applicable mathematical algorithms to insert them into his software program. Initially, he generated more than 100 graphic diagrams which were based on $\sim 100,000$ data of glucose, HbA1C, carbs/sugar input, exercise, and weather temperature. By using his two eyes and brain (natural intelligence) to visually discover and study some hidden patterns in those $100+$ graphic diagrams, he could extract certain odd phenomena, hidden evidence, and possible clues. Eventually, he has developed a set of mathematical equations or formulas that are not only capable to describe but also predict those physical behaviors and characteristics of Sensor glucoses.

\section{Results}

Figure 1: shows the total period of 17-months (527 days) from $5 / 5 / 2018$ to $10 / 13 / 2019$, including this special four months sub-period of "14-day sensor device reliability" from 6/2/2019 $-10 / 13 / 2019$. This diagram includes glucoses (both Sensor and Finger), carbs/sugar intake amount, and post-meal walking steps.

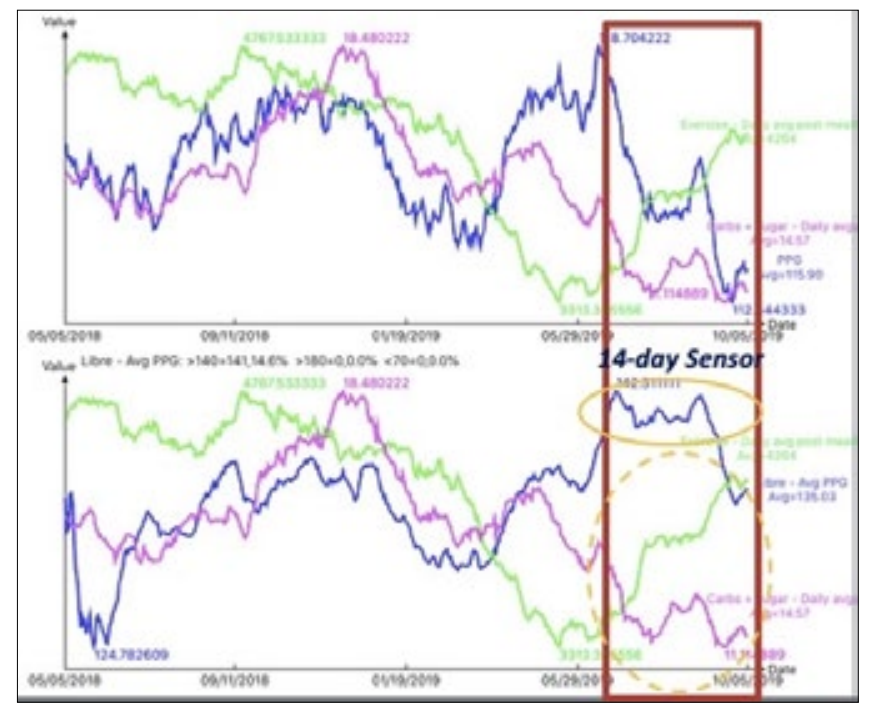

Figure 1: Overview of 17-months period (5/5/2018 $10 / 5 / 2019)$ and 3 months sub-period of 14-day sensor device $(6 / 30 / 2019-10 / 5 / 2019)$ 
Figure 2 displays the comparison between Finger PPG and 14day Sensor PPG.

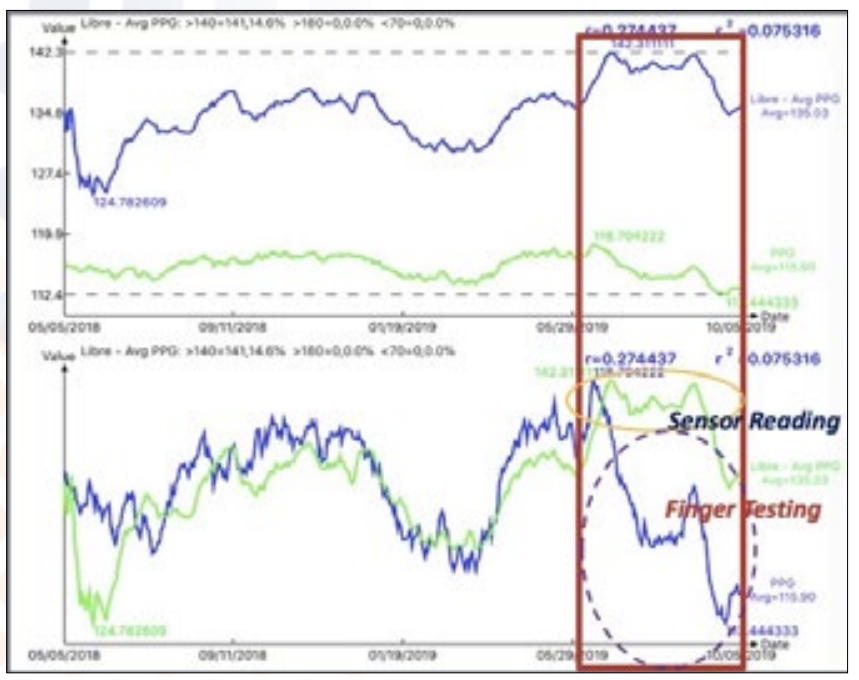

Figure 2: Comparison of Finger PPG and 14-day Sensor PPG (Sub-period of 14-day sensor device)

Figure 3 further illustrates a much stronger pattern similarity between Finger PPG and Daily HbA1C. The declined Finger PPG matched with the declined pattern of HbA1C (from both daily mathematical $\mathrm{HbA} 1 \mathrm{C}$ curve and two lab-tested discrete HbA1C data).

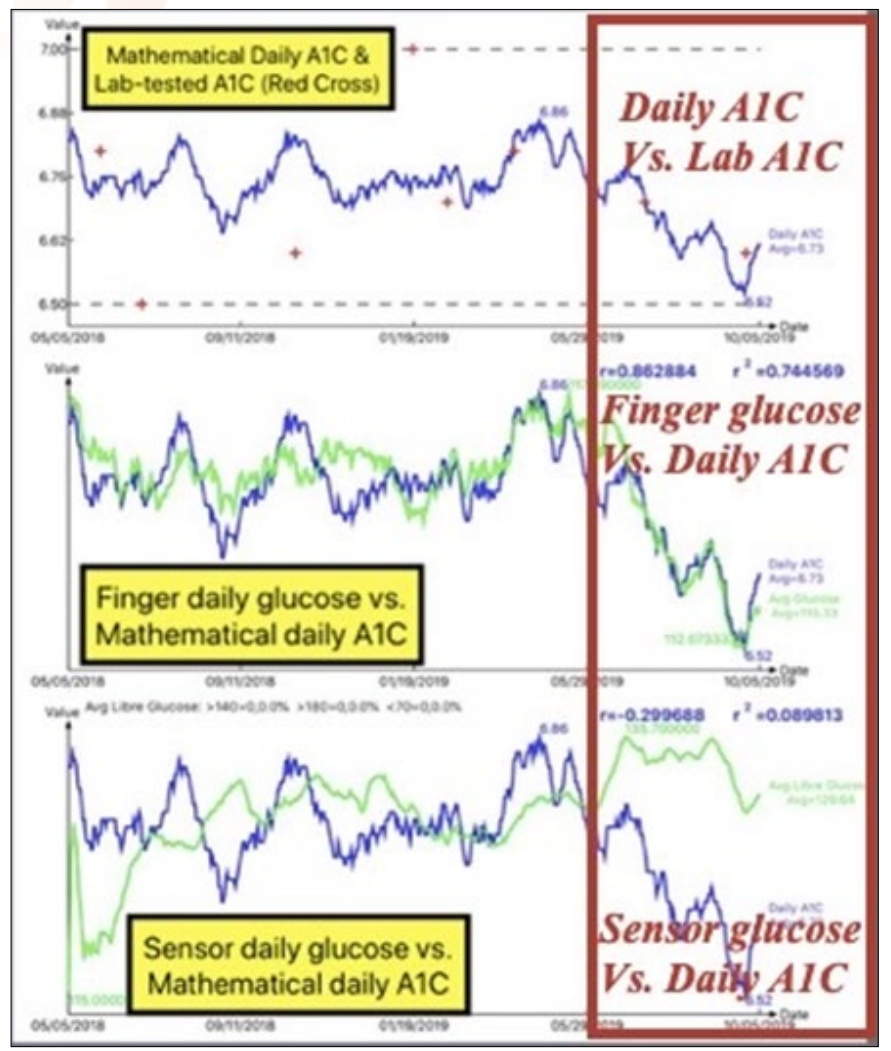

Figure 3: Finger PPG vs. HbA1C and Sensor PPG vs. HbA1C shows strong pattern similarity between Finger glucose and Daily HbA1C
Figure 4 shows detailed comparison between 10-day Sensor sub-period (5/5/2018 - 6/2/2019) and 14-day Sensor sub-period (6/2/2019 - 10/13/2019). The sub-period of "before 6/2/2019" has a very high correlation coefficients between Sensor glucose and Finger glucose $(89 \%)$ which means a very strong pattern similarity. The sub-period of "after $6 / 2 / 2019$ " has a lower correlation between Finger and Sensor (43\%) which means a weaker pattern similarity. However, as the author suspected in early July of 2019, the average glucose difference between 14days (higher at $24 \mathrm{mg} / \mathrm{dL}$ ) and 10-days (lower at $18 \mathrm{mg} / \mathrm{dL}$ ) is $6 \mathrm{mg} / \mathrm{dL}$. It should be pointed out that the gap between Sensor PPG vs. Finger PPG for sub-period of "after 6/2/2019" is much wider (within the central region of this sub-period, $\sim 80 \%$ of total area).

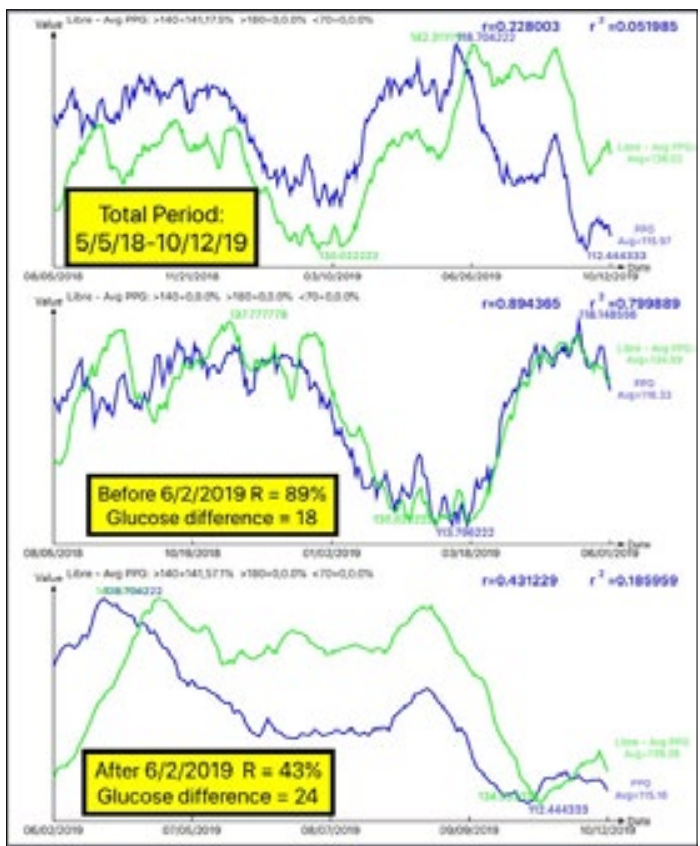

Figure 4: High R between Finger and Sensor before 6/2/2019 and low $\mathrm{R}$ after $6 / 2 / 19$

As shown in Figure 5, during this sub-period of "after 6/2/2019", both of the Finger PPG and Sensor PPG patterns are obviously following the expected combined effect of increased exercise and near-constant carbs intake. It should be noted that, the Sensor PPG curve's plateau height is still much higher than the Finger PPG curve' plateau.

In summary, the author has some questions regarding the general reliability of the new 14-day sensor device. Due to the observed similar patterns between waveforms of Finger vs. both 10-day and 14-day Sensors, the author hypothesized that the 14-day Sensor design has followed the same design as the 10-day Sensor. However, based on the observation of different glucose plateaus heights, the author detected that the 14-day Sensor's glucose data alignment may have some built-in error.

Due to the author's fear of rising glucose readings from this device, during this "after" sub-period, he has been more conscious on following the principle of eating less and walking more. As a result, his Finger PPG glucose curve has shown 
a sharp decline which caused the reduction of his $\mathrm{HbA1C}$ as well.

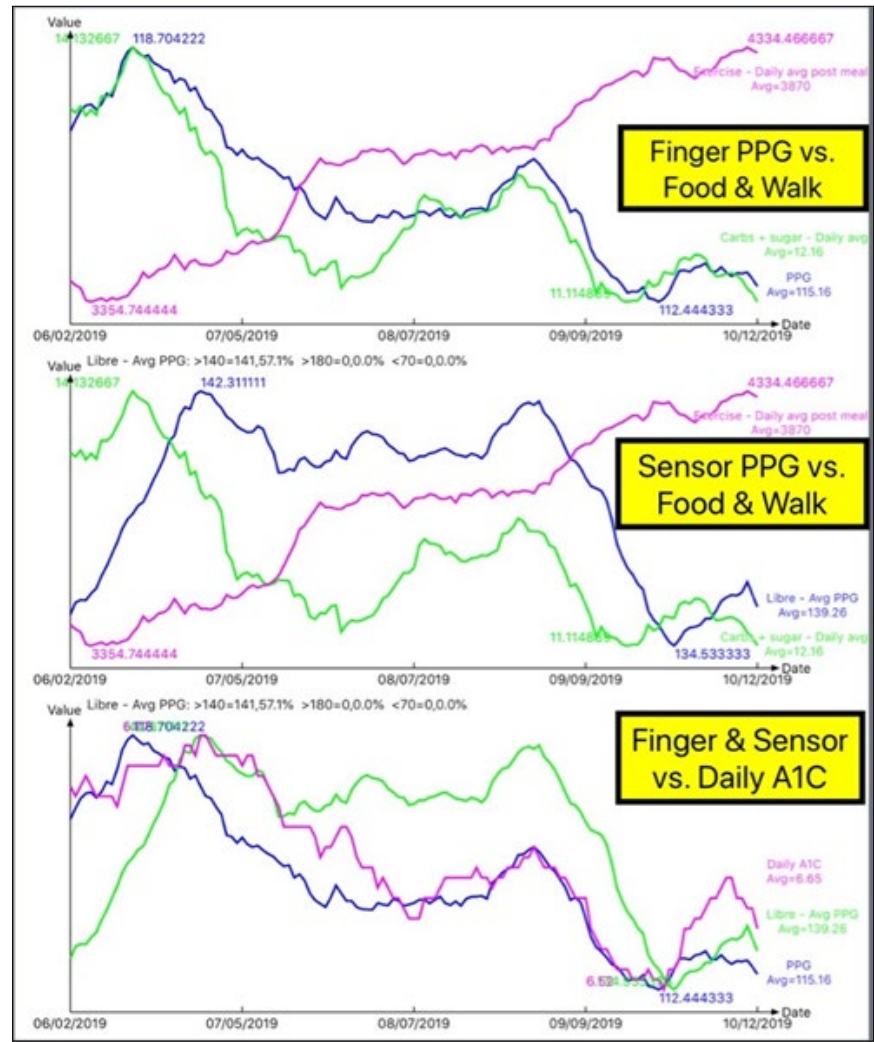

Figure 5: detailed analysis of Sensor PPG resulted from both carbs intake and walking during sub-period of 14-day Sensor Device.

It is interesting to see some specific reliability issues in Figure 6 . The top diagram's digital reading of glucose at $13: 36$ was 176 $\mathrm{mg} / \mathrm{dL}$ (right side); however, its indicated glucose curve was around $120 \mathrm{mg} / \mathrm{dL}$ (left side) at that time instant. Of course, this could be caused from a kind of "hand-shake" problem between data and graph. The bottom two diagrams show early morning fasting glucoses of 10/13/2019 at 54 and $64 \mathrm{mg} / \mathrm{dL}$, while Finger glucoses at 89 and $108 \mathrm{mg} / \mathrm{dL}$. These fasting glucose differences were extremely large ( 35 and $44 \mathrm{mg} / \mathrm{dL}$ lower). Obviously, these examples further indicated that there are some reliability issues associated with this 14-day sensor device.

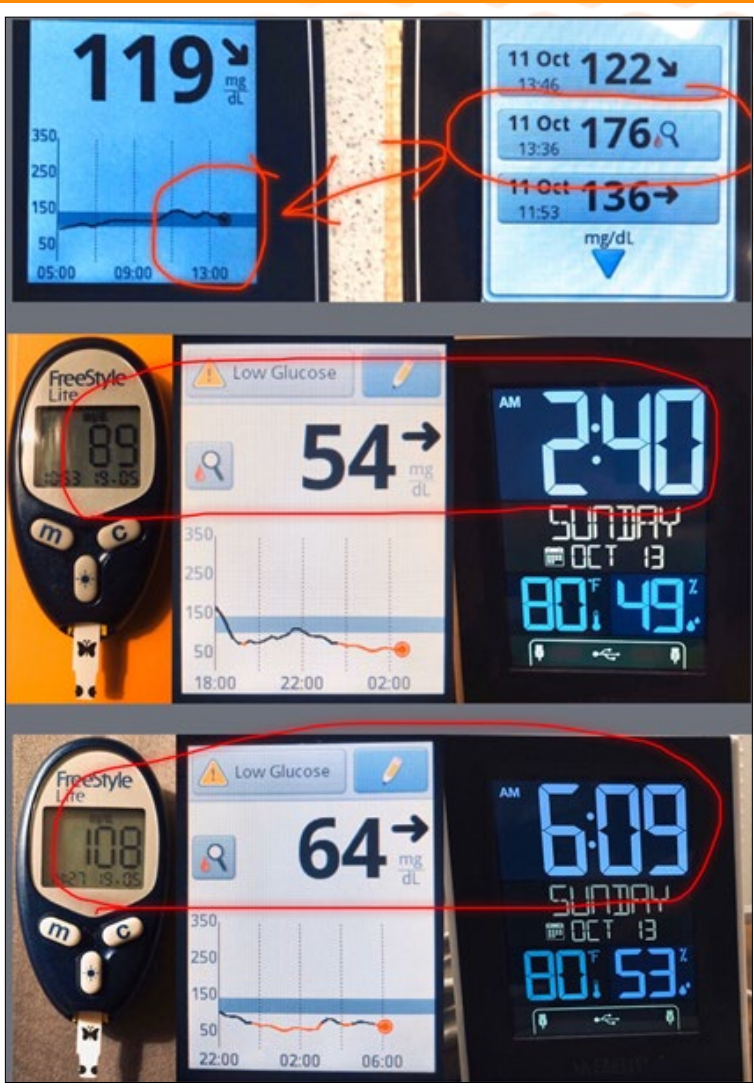

Figure 6: Mismatch of glucose digital reading (right side) and glucose curve (left side)

In Figure 7, there was an additional overall reliability issue of the Sensor data patterns. The author noticed his Sensor glucose data seem to be lower near the end of the device's 14-day period. After waiting for over 90 days, he started to reassemble these special data and then calculate the averaged glucose near-the-end of 14-day lifespan. He then found the averaged near-the-end glucose of 8 days is $115.0 \mathrm{mg} / \mathrm{dL}(88 \%)$ in comparison with the average daily glucose of $129.9 \mathrm{mg} / \mathrm{dL}$ $(100 \%)$ based on the total 106 days $(6 / 30 / 2019-10 / 13 / 2019$ using the same reader). There was an averaged $12 \%$ or $15 \mathrm{mg} /$ dL lower glucose value. The author suspected that the reason for this kind of "flaw" could be related to the device's chemical process.

For a comparative purpose, there are 24 days $(22.6 \%$ of 106 days) with glucoses greater than $140 \mathrm{mg} / \mathrm{dL}$. These "high glucose" days averaged glucose is $147 \mathrm{mg} / \mathrm{dL}$ which is $17 \mathrm{mg}$ / dL (13\%) higher than the averaged glucose of total 106 days at $129.9 \mathrm{mg} / \mathrm{dL}$ (use this as $100 \%$ ). 


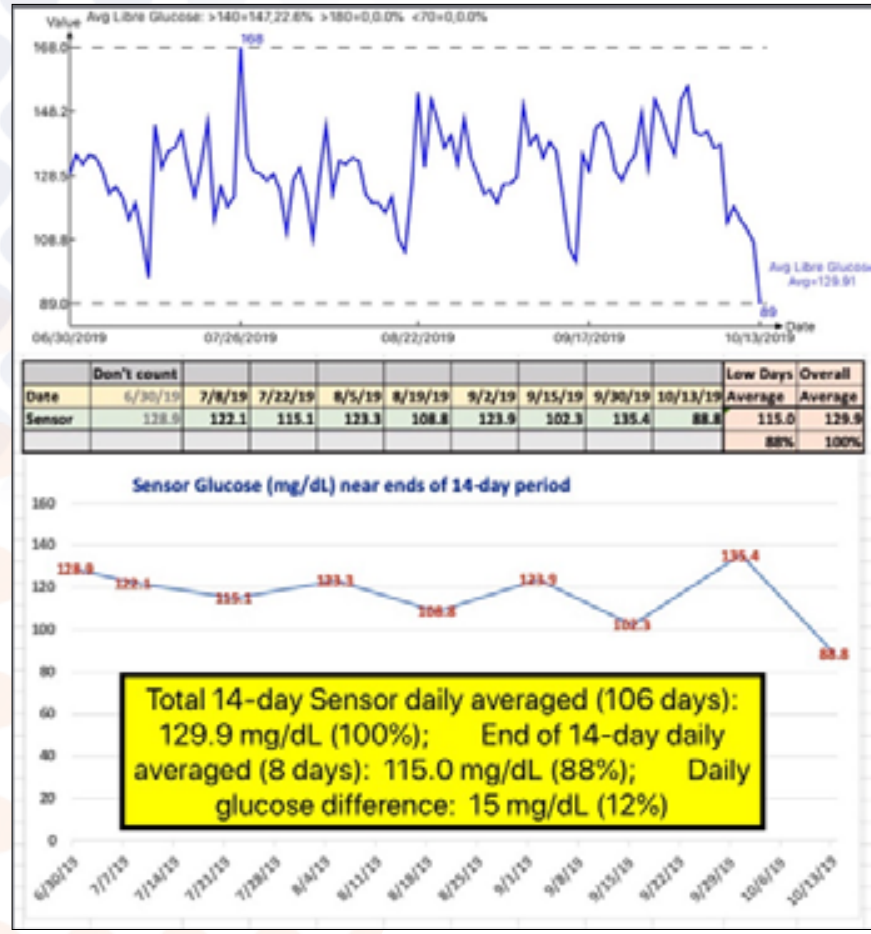

Figure 7: Lower daily glucoses near the end of 14-day Sensor period.

\section{Conclusions}

Although this 14-day sensor device still has some merits, its glucose readings lack the necessary precision. If a diabetes patient uses this kind of sensor device to determine the timing and dosage of the insulin injection, then it is important to pay closer attention to the precision and reliability of this kind of glucose measuring device. If this 14-day sensor device consistently shows higher glucose than its actual glucose state, then the incorrect administration of insulin may result in serious problems such as overdosage leading to insulin shock.

From the 14-day sensor device case study, the author believes that, within the scope of endocrinology and, up to now, those existing research mythologies and certain medical device designs lack the necessary precision and accuracy like some other branches of natural science research. The author has received a thorough training in both academic studies and professional experiences in the areas of mathematics, physics, engineering, and computer science, excluding biology and chemistry. Instead of using the biochemical approach to research medicine issues, he used physical phenomena observation and interpretation followed by mathematical derivation and proof. In 2010, he entered into the medical research field of diabetes and its complications in order to save his own life. When comparing his previous design experiences in space shuttle and semiconductors, he could not help but wonder what would have happened to his previous job assignments if he applied this kind of "existing internal medicine's research methodology" on those jobs? His intentions are not to deliberately offend his medical research scientists in any way. He is merely suggesting that a different but more precise approach could reveal additional hidden facts or discover some more useful conclusions regarding these important but dangerous metabolic and endocrinological diseases [1-6].

\section{References}

1. Hsu, Gerald C (2018) Using Math-Physical Medicine to Control T2D via Metabolism Monitoring and Glucose Predictions. Journal of Endocrinology and Diabetes 1(1): 1-6.

2. Hsu, Gerald C (2018) Using Signal Processing Techniques to Predict PPG for T2D. International Journal of Diabetes \& Metabolic Disorders 3(2): 1-3.

3. Hsu, Gerald C (2018) Using Math-Physical Medicine and Artificial Intelligence Technology to Manage Lifestyle and Control Metabolic Conditions of T2D. International Journal of Diabetes \& Its Complications 2(3): 1-7.

4. Hsu, Gerald C (2018) Using Math-Physical Medicine to Analyze Metabolism and Improve Health Conditions. Video presented at the meeting of the 3rd International Conference on Endocrinology and Metabolic Syndrome 2018, Amsterdam, Netherlands.

5. Hsu, Gerald C (2018) Using Math-Physical Medicine to Study the Risk Probability of having a Heart Attack or Stroke Based on Three Approaches, Medical Conditions, Lifestyle Management Details, and Metabolic Index. EC Cardiology 5(12): 1-9.

6. Hsu, Gerald C (2018) Using Signal Processing Techniques to Predict PPG for T2D. International Journal of Diabetes \& Metabolic Disorders 3(2): 1-3.
Copyright: (C2020 Gerald C. Hsu. This is an open-access article distributed under the terms of the Creative Commons Attribution License, which permits unrestricted use, distribution, and reproduction in any medium, provided the original author and source are credited. 